\title{
Hyperbolically convex functions II
}

\author{
by William Ma (Williamsport, Penn.) and \\ David Minda (Cincinnati, Ohio)
}

\begin{abstract}
Unlike those for euclidean convex functions, the known characterizations for hyperbolically convex functions usually contain terms that are not holomorphic. This makes hyperbolically convex functions much harder to investigate. We give a geometric proof of a two-variable characterization obtained by Mejia and Pommerenke. This characterization involves a function of two variables which is holomorphic in one of the two variables. Various applications of the two-variable characterization result in a number of analogies with the classical theory of euclidean convex functions. In particular, we obtain a uniform upper bound on the Schwarzian derivative. We also obtain the sharp lower bound on $\left|f^{\prime}(z)\right|$ for all $z$ in the unit disk, and the sharp upper bound on $\left|f^{\prime}(z)\right|$ when $|z| \leq \sqrt{2}-1$.
\end{abstract}

1. Introduction. A holomorphic and univalent function $f$ defined on the unit disk $\mathbb{D}=\{z:|z|<1\}$ is called hyperbolically convex if its image $f(\mathbb{D})$ is a hyperbolically convex subset of $\mathbb{D}$. A subregion $\Omega$ of $\mathbb{D}$ is called hyperbolically convex (relative to hyperbolic geometry on $\mathbb{D}$ ) if for all points $a, b \in \Omega$ the arc of the hyperbolic geodesic in $\mathbb{D}$ connecting $a$ and $b$ (the arc of the circle joining $a$ and $b$ which is orthogonal to the unit circle) lies in $\Omega$. The classical theory of euclidean convex functions $f$ defined on $\mathbb{D}$ is well developed partially because of the simple characterization $\operatorname{Re}\left\{1+z f^{\prime \prime}(z) / f^{\prime}(z)\right\}>0$. Much less is known about hyperbolically convex functions. In [3], we established a number of characterizations for hyperbolically convex functions. For example,

$$
\left|\frac{\left(1-|z|^{2}\right) f^{\prime \prime}(z)}{2 f^{\prime}(z)}+\frac{\left(1-|z|^{2}\right) \overline{f(z)} f^{\prime}(z)}{1-|f(z)|^{2}}-\bar{z}\right| \leq 1-\left(\frac{\left(1-|z|^{2}\right)\left|f^{\prime}(z)\right|}{1-|f(z)|^{2}}\right)^{2} .
$$

Even though we were able to derive sharp upper and lower bounds on $\left(1-|z|^{2}\right)\left|f^{\prime}(z)\right| /\left(1-|f(z)|^{2}\right)$ and $|f(z)|$ from this characterization, it is not easy to use, partly because it contains the nonholomorphic term $\left(1-|z|^{2}\right) \overline{f(z)} f^{\prime}(z) /\left(1-|f(z)|^{2}\right)$.

1991 Mathematics Subject Classification: Primary 30C45, 30C50.

Key words and phrases: hyperbolic convexity, two-variable characterization, Schwarzian derivative, distortion theorem. 
Another characterization for hyperbolically convex functions we had in [3] is

$$
\operatorname{Re}\left\{1+\frac{z f^{\prime \prime}(z)}{f^{\prime}(z)}+\frac{2 \overline{f(z)} z f^{\prime}(z)}{1-|f(z)|^{2}}\right\}>0 \quad(z \in \mathbb{D}) .
$$

This is similar to the well known characterization $\operatorname{Re}\left\{1+z f^{\prime \prime}(z) / f^{\prime}(z)\right\}>0$ for euclidean convex functions. Again, the term $2 \overline{f(z)} z f^{\prime}(z) /\left(1-|f(z)|^{2}\right)$ is not holomorphic in $\mathbb{D}$.

In this paper, we first give a geometric proof of a two-variable characterization for hyperbolically convex functions obtained by Mejia and Pommerenke [6]; this characterization is easier to use in certain cases since it is holomorphic in one of the variables. We provide several applications of this two-variable characterization.

First, we generalize the result that $f(z)^{2} / z$ is starlike in $\mathbb{D}$ if $f(z)$ is hyperbolically convex with $f(0)=0$, obtained recently by Mejia and Pommerenke [6]. They used this fact to give a simpler proof of the first characterization we mentioned earlier.

Then we prove that $\operatorname{Re}\left\{a_{2} f(z) / \alpha^{2}\right\}>-1, z \in \mathbb{D}$, if $f(z)=\alpha z+$ $a_{2} z^{2}+\ldots$ is hyperbolically convex. A similar result holds for euclidean convex functions [2].

We also use the two-variable characterization to get a uniform upper bound on the Schwarzian derivative of hyperbolically convex functions.

Finally, we obtain the sharp lower bound on $\left|f^{\prime}(z)\right|$ for all $z \in \mathbb{D}$, and the sharp upper bound on $\left|f^{\prime}(z)\right|$ when $|z| \leq \sqrt{2}-1$ by using the sharp growth theorem given in [3]. This sharp upper bound was first obtained by Mejia, Pommerenke and Vasil'ev [5].

2. Preliminaries. The hyperbolic metric on the unit disk $\mathbb{D}$ is given by $\lambda_{\mathbb{D}}(z)|d z|=|d z| /\left(1-|z|^{2}\right)$. The unique hyperbolic geodesic in $\mathbb{D}$ connecting $a$ and $b$ is the arc $\gamma$ of the circle through $a$ and $b$ that is orthogonal to the unit circle.

As in [3], we use two differential operators for holomorphic functions $f: \mathbb{D} \rightarrow \mathbb{D}$. Set

and

$$
D_{h 1} f(z)=\frac{\left(1-|z|^{2}\right) f^{\prime}(z)}{1-|f(z)|^{2}}
$$

$$
D_{h 2} f(z)=\frac{\left(1-|z|^{2}\right)^{2} f^{\prime \prime}(z)}{1-|f(z)|^{2}}+\frac{2\left(1-|z|^{2}\right)^{2} \overline{f(z)} f^{\prime}(z)^{2}}{\left(1-|f(z)|^{2}\right)^{2}}-\frac{2 \bar{z}\left(1-|z|^{2}\right) f^{\prime}(z)}{1-|f(z)|^{2}} .
$$

The use of these two differential operators simplifies many formulas. For more information about these two operators, see [4]. They satisfy the invariance property $\left|D_{h j}(R \circ f \circ S)\right|=\left|D_{h j} f\right| \circ S(j=1,2)$ which holds for 
all conformal automorphisms $R, S$ of $\mathbb{D}$. Note that $D_{h 1} f(0)=f^{\prime}(0)$ and $D_{h 2} f(0)=f^{\prime \prime}(0)$ if $f(0)=0$. Also, $D_{h 1} f(a)=f_{a}^{\prime}(0), D_{h 2} f(a)=f_{a}^{\prime \prime}(0)$ if

$$
f_{a}(z)=\frac{f((z+a) /(1+\bar{a} z))-f(a)}{1-\overline{f(a)} f((z+a) /(1+\bar{a} z))} .
$$

Recall that if $f(z)$ is holomorphic and locally univalent in $\mathbb{D}$, then the Schwarzian derivative of $f(z)$ is

$$
S_{f}(z)=\frac{f^{\prime \prime \prime}(z)}{f^{\prime}(z)}-\frac{3}{2}\left(\frac{f^{\prime \prime}(z)}{f^{\prime}(z)}\right)^{2} .
$$

An important example of a hyperbolically convex function is (see [3])

$$
\begin{aligned}
k_{\alpha}(z) & =\frac{2 \alpha z}{1-z+\sqrt{(1-z)^{2}+4 \alpha^{2} z}} \\
& =\alpha z+\alpha\left(1-\alpha^{2}\right) z^{2}+\alpha\left(1-\alpha^{2}\right)\left(1-2 \alpha^{2}\right) z^{3}+\ldots,
\end{aligned}
$$

which maps $\mathbb{D}$ conformally onto the hyperbolic half-plane

$$
k_{\alpha}(\mathbb{D})=\mathbb{D} \backslash\left\{w:\left|w+\frac{1}{\alpha}\right| \leq \frac{1}{\alpha} \sqrt{1-\alpha^{2}}\right\} .
$$

Next, we prove a lemma about the class $\mathcal{P}$ that we need later. Here $\mathcal{P}$ consists of the holomorphic functions $p(z)$ in $\mathbb{D}$ with $p(0)=1$ and $\operatorname{Re}\{p(z)\}>0$.

Lemma 2.1. Let $p(z) \in \mathcal{P}$. Then we have the sharp estimates: for $0<$ $|z|<1$,

$$
\left|2 z p^{\prime}(z)+1-p(z)^{2}\right|+\left|p(z)-\frac{1+|z|^{2}}{1-|z|^{2}}\right|^{2} \leq\left(\frac{2|z|}{1-|z|^{2}}\right)^{2},
$$

and for $z=0$,

$$
\left|p^{\prime \prime}(0)-p^{\prime}(0)^{2}\right|+\left|p^{\prime}(0)\right|^{2} \leq 4
$$

Proof. Let

$$
w(z)=\frac{p(z)-1}{p(z)+1}
$$

and $h(z)=w(z) / z$. Note that $w(z)$ is holomorphic in $\mathbb{D}$ with $w(0)=0$ and $|w(z)|<1$ in $\mathbb{D}$. Therefore, $h(z)$ is also holomorphic on $\mathbb{D}$ with $h(0)=w^{\prime}(0)$ and $|h(z)| \leq 1$ for $z \in \mathbb{D}$. The Schwarz-Pick Lemma gives

or

$$
\frac{\left|h^{\prime}(z)\right|}{1-|h(z)|^{2}} \leq \frac{1}{1-|z|^{2}}
$$

$$
\left(1-|z|^{2}\right)\left|h^{\prime}(z)\right|+|h(z)|^{2} \leq 1 .
$$

Since

$$
h(0)=w^{\prime}(0)=\frac{1}{2} p^{\prime}(0)
$$


and

the inequality $(2.2)$ follows.

$$
h^{\prime}(0)=\frac{1}{2} w^{\prime \prime}(0)=\frac{1}{4}\left(p^{\prime \prime}(0)-p^{\prime}(0)^{2}\right),
$$

For $z \neq 0$, we get

$$
\left(1-|z|^{2}\right)\left|z w^{\prime}(z)-w(z)\right|+|w(z)|^{2} \leq|z|^{2} .
$$

The definition of $w(z)$ together with

then yields

$$
\frac{2 z p^{\prime}(z)+1-p(z)^{2}}{(p(z)+1)^{2}}=z w^{\prime}(z)-w(z)
$$

$$
\left(1-|z|^{2}\right)\left|2 z p^{\prime}(z)+1-p(z)^{2}\right|+|p(z)-1|^{2} \leq|z|^{2}|p(z)+1|^{2} .
$$

Because

$$
|p(z)-1|^{2}-|z|^{2}|p(z)+1|^{2}=\left(1-|z|^{2}\right)\left|p(z)-\frac{1+|z|^{2}}{1-|z|^{2}}\right|^{2}-\frac{4|z|^{2}}{1-|z|^{2}},
$$

we now obtain (2.1).

Clearly, equality holds in $(2.2)$ for $p(z)=(1+z) /(1-z)$ and in (2.1) for $p(z)=(1+z) /(1-z)$ when $z \in(-1,1)$.

\section{A two-variable characterization of hyperbolically convex}

functions. Sheil-Small [8] and Suffridge [9] obtained a useful two-variable characterization

$$
\operatorname{Re}\left\{\frac{2 z f^{\prime}(z)}{f(z)-f(a)}-\frac{z+a}{z-a}\right\}>0
$$

for euclidean convex functions, which implies that $\operatorname{Re}\left\{1+z f^{\prime \prime}(z) / f^{\prime}(z)\right\}>0$ and

$$
F(z)=\frac{z a}{f(a)} \frac{f(z)-f(a)}{z-a}
$$

is starlike of order $1 / 2$ for every $a \in \mathbb{D}$. We now give a geometric proof of a similar two-variable characterization for hyperbolically convex functions. This characterization was obtained by Mejia and Pommerenke. Their expression is not written similarly as the expression above for euclidean convex functions and their proof is rather different from what we have here (see [5, Cor. 3.4]). This characterization is easier to use in some cases as it involves a holomorphic function in $z$.

Theorem 3.1 (Mejia-Pommerenke). Let $f(z)$ be holomorphic and locally univalent in $\mathbb{D}$ with $f(\mathbb{D}) \subset \mathbb{D}$. Then $f(z)$ is hyperbolically convex if and only if

$$
\operatorname{Re}\left\{\frac{2 z f^{\prime}(z)}{f(z)-f(a)}-\frac{z+a}{z-a}+\frac{2 \overline{f(a)} z f^{\prime}(z)}{1-\overline{f(a)} f(z)}\right\}>0 \quad(z \in \mathbb{D}, a \in \mathbb{D}) .
$$


Pr o of. First, we assume that $f(z)$ is hyperbolically convex. The hyperbolic geodesic $\gamma$ from $A$ to $B \in \mathbb{D}$ can be expressed by

$$
w(t)=\frac{t(B-A) /(1-\bar{A} B)+A}{1+t \bar{A}(B-A) /(1-\bar{A} B)}, \quad 0 \leq t \leq 1 .
$$

At $A$, the tangent vector to $\gamma$ is

$$
w^{\prime}(0)=\frac{B-A}{1-\bar{A} B}\left(1-|A|^{2}\right) .
$$

For $a=r e^{i \theta} \in \mathbb{D}$ and $z=r e^{i \varphi}, \theta<\varphi<\theta+2 \pi$, we consider the hyperbolic geodesic $\gamma_{\varphi}$ connecting $f(a)$ and $f(z)$. The tangent vector to $\gamma_{\varphi}$ at $f(a)$ is

$$
w_{\varphi}^{\prime}(0)=\frac{f\left(r e^{i \varphi}\right)-f(a)}{1-\overline{f(a)} f\left(r e^{i \varphi}\right)}\left(1-|f(a)|^{2}\right) .
$$

Since $f(|z|=r)$ is a hyperbolically convex curve,

$$
L(\varphi)=\arg \left\{w_{\varphi}^{\prime}(0)\right\}=\arg \left\{\frac{f\left(r e^{i \varphi}\right)-f(a)}{1-\overline{f(a)} f\left(r e^{i \varphi}\right)}\left(1-|f(a)|^{2}\right)\right\}
$$

is increasing in $(\theta, \theta+2 \pi)$ and therefore

$$
\begin{aligned}
L^{\prime}(\varphi) & =\frac{\partial}{\partial \varphi} \arg \left[f\left(r e^{i \varphi}\right)-f(a)\right]-\frac{\partial}{\partial \varphi} \arg \left[1-\overline{f(a)} f\left(r e^{i \varphi}\right)\right] \\
& =\operatorname{Im}\left\{\frac{f^{\prime}\left(r e^{i \varphi}\right) i r e^{i \varphi}}{f\left(r e^{i \varphi}\right)-f(a)}\right\}+\operatorname{Im}\left\{\frac{\overline{f(a)} f^{\prime}\left(r e^{i \varphi}\right) i r e^{i \varphi}}{1-\overline{f(a)} f\left(r e^{i \varphi}\right)}\right\} \\
& =\operatorname{Re}\left\{\frac{z f^{\prime}(z)}{f(z)-f(a)}+\frac{\overline{f(a)} z f^{\prime}(z)}{1-\overline{f(a)} f(z)}\right\} \geq 0 .
\end{aligned}
$$

Let

$$
p(z, a)=\frac{2 z f^{\prime}(z)}{f(z)-f(a)}-\frac{z+a}{z-a}+\frac{2 \overline{f(a)} z f^{\prime}(z)}{1-\overline{f(a)} f(z)} .
$$

As $\operatorname{Re}\{(z+a) /(z-a)\}=0$ for $|z|=|a|=r, z \neq a$ we conclude that

$$
\operatorname{Re}\{p(z, a)\} \geq 0 \quad(|z|=|a|=r)
$$

for $z=a$ this follows from continuity. Because $\operatorname{Re}\{p(z, a)\}$ is harmonic in both $z$ and $a$, the maximum principle yields $\operatorname{Re}\{p(z, a)\} \geq 0$ for $|z|<r$ and $|a|<r$. If we let $\rightarrow 1$ we see that $\operatorname{Re}\{p(z, a)\} \geq 0$ for $z, a \in \mathbb{D}$. The maximum principle for harmonic functions again implies $\operatorname{Re}\{p(z, a)\}>0$ for $z, a \in \mathbb{D}$ since $p(0, a)=1$, which means that $\operatorname{Re}\{p(z, a)\}$ cannot be identically 0 .

Now we show that if $f$ satisfies the two-variable inequality (3.1), then

$$
\operatorname{Re}\left\{1+\frac{a f^{\prime \prime}(a)}{f^{\prime}(a)}+\frac{2 \overline{f(a)} a f^{\prime}(a)}{1-|f(a)|^{2}}\right\}>0
$$


for all $a \in \mathbb{D}$ since this characterizes hyperbolically convex functions [3]. The assumption is that $\operatorname{Re}\{p(z, a)\}>0$ for all $z, a \in \mathbb{D}$. Since

$$
p(a, a)=1+\frac{a f^{\prime \prime}(a)}{f^{\prime}(a)}+\frac{2 \overline{f(a)} a f^{\prime}(a)}{1-|f(a)|^{2}},
$$

the proof is complete.

As we mentioned earlier, we cannot easily get properties for hyperbolically convex functions from (1.1) since it has a term that is not holomorphic. With the help of Theorem 3.1, this difficulty is overcome in some cases. To illustrate our point, we show in the proof of the following corollary how Theorem 3.1 can be used to prove that the nonholomorphic function

still satisfies

$$
p(z)=1+\frac{z f^{\prime \prime}(z)}{f^{\prime}(z)}+\frac{2 \overline{f(z)} z f^{\prime}(z)}{1-|f(z)|^{2}}
$$

$$
\left|p(z)-\frac{1+|z|^{2}}{1-|z|^{2}}\right| \leq \frac{2|z|}{1-|z|^{2}},
$$

which is a well known result for $\mathcal{P}$. We state the result for hyperbolically convex functions; a similar characterization with strict inequality was given in [3], and its proof was quite different from what we have here.

Corollary 3.2. Suppose $f(z)$ is holomorphic and locally univalent in $\mathbb{D}$ with $f(\mathbb{D}) \subset \mathbb{D}$. Then $f(z)$ is hyperbolically convex if and only if

$$
\left|\frac{D_{h 2} f(z)}{D_{h 1} f(z)}\right| \leq 2 \quad(z \in \mathbb{D}) .
$$

Proof. First, we assume that $f(z)$ is hyperbolically convex. It is enough to prove the inequality at any fixed $a \in \mathbb{D}$. Theorem 3.1 implies that $p(z, a)$ given in (3.2) has positive real part as a function of $z$. Thus, $p(z, a)$ belongs to $\mathcal{P}$ as a function of $z$ and

$$
\left|p(z, a)-\frac{1+|z|^{2}}{1-|z|^{2}}\right| \leq \frac{2|z|}{1-|z|^{2}} .
$$

As

we obtain

$$
p(a, a)=1+\frac{a f^{\prime \prime}(a)}{f^{\prime}(a)}+\frac{2 \overline{f(a)} a f^{\prime}(a)}{1-|f(a)|^{2}}
$$

$$
\left|1+\frac{a f^{\prime \prime}(a)}{f^{\prime}(a)}+\frac{2 \overline{f(a)} a f^{\prime}(a)}{1-|f(a)|^{2}}-\frac{1+|a|^{2}}{1-|a|^{2}}\right| \leq \frac{2|a|}{1-|a|^{2}} .
$$

The preceding inequality is equivalent to

$$
\left|\frac{D_{h 2} f(a)}{D_{h 1} f(a)}\right| \leq 2 \text {. }
$$


By reversing the steps above, we see that $\left|D_{h 2} f(a) / D_{h 1} f(a)\right| \leq 2$ implies

Thus

$$
\left|p(a, a)-\frac{1+|a|^{2}}{1-|a|^{2}}\right| \leq \frac{2|a|}{1-|a|^{2}} .
$$

$$
\operatorname{Re}\{p(a, a)\} \geq \frac{1-|a|}{1+|a|}>0 .
$$

4. Some applications of the two-variable characterization. Recall that a holomorphic and locally univalent function $f(z)$ in $\mathbb{D}$ with $f(0)=$ $f^{\prime}(0)-1=0$ is called starlike of order $\beta$ if $\operatorname{Re}\left\{z f^{\prime}(z) / f(z)\right\}>\beta$. Mejia and Pommerenke [6] showed that $f(z) / f^{\prime}(0)$ is starlike of order $1 / 2$ if $f(z)$ is hyperbolically convex with $f(0)=0$. This is the special case of the following result when $a=0$.

THEOREM 4.1. If $f(z)$ is hyperbolically convex with $f(0)=0$, then for every $a \in \mathbb{D}$,

is starlike of order $1 / 2$.

$$
F_{a}(z)=\frac{z a}{f(a)} \frac{f(z)-f(a)}{(z-a)(1-\overline{f(a)} f(z))}
$$

Proof. $F_{a}(z)$ is starlike of order $1 / 2$ if and only if

As

$$
\operatorname{Re}\left\{\frac{2 z F_{a}^{\prime}(z)}{F_{a}(z)}-1\right\}>0
$$

we have

$$
\frac{z F_{a}^{\prime}(z)}{F_{a}(z)}=\frac{z f^{\prime}(z)}{f(z)-f(a)}-\frac{a}{z-a}+\frac{\overline{f(a)} z f^{\prime}(z)}{1-\overline{f(a)} f(z)},
$$

$$
\frac{2 z F_{a}^{\prime}(z)}{F_{a}(z)}-1=\frac{2 z f^{\prime}(z)}{f(z)-f(a)}-\frac{z+a}{z-a}+\frac{2 \overline{f(a)} z f^{\prime}(z)}{1-\overline{f(a)} f(z)} .
$$

Theorem 3.1 then implies the desired inequality.

Mejia and Pommerenke [6] showed that for hyperbolically convex functions $f(z)$ with $f(0)=0$,

$$
\operatorname{Re}\left\{\frac{a}{f(a)} \frac{f(z)-f(a)}{z-a}\right\}>\frac{1}{2} .
$$

As a corollary of Theorem 4.1, we now state a similar result. We use the fact that $\operatorname{Re}\{F(z) / z\}>1 / 2$ if $F(z)$ is starlike of order $1 / 2$ (see [7, p. 49]).

COROLlary 4.2. If $f(z)$ is hyperbolically convex with $f(0)=0$, then for every $a \in \mathbb{D}$,

$$
\operatorname{Re}\left\{\frac{a}{f(a)} \frac{f(z)-f(a)}{(z-a)(1-\overline{f(a)} f(z))}\right\}>\frac{1}{2} \quad(z \in \mathbb{D}) .
$$


Corollary 4.3. If $f(z)$ is hyperbolically convex with $f(0)=0$, then for every $a \in \mathbb{D}$,

is starlike in $\mathbb{D}$.

$$
\frac{F_{a}(z)^{2}}{z}=\frac{z a^{2}}{f(a)^{2}} \frac{(f(z)-f(a))^{2}}{(z-a)^{2}(1-\overline{f(a)} f(z))^{2}}
$$

When $a=0$, this result is due to Mejia and Pommerenke [6].

As another application of Theorem 3.1, we provide a lower bound on $\operatorname{Re}\left\{a_{2} f(z) / \alpha^{2}\right\}$ for hyperbolically convex functions.

THEOREM 4.4. Let $f(z)=\alpha z+a_{2} z^{2}+\ldots$ be hyperbolically convex. Then

$$
\operatorname{Re}\left\{\frac{a_{2} f(z)}{\alpha^{2}}\right\}>-1 \quad(z \in \mathbb{D}) .
$$

Proof. For any $a \in \mathbb{D}$, we define $p(z)$ by

$$
p(z)=\frac{2 z f^{\prime}(z)}{f(z)-f(a)}-\frac{z+a}{z-a}+\frac{2 \overline{f(a)} z f^{\prime}(z)}{1-\overline{f(a)} f(z)},
$$

which belongs to $\mathcal{P}$ by Theorem 3.1. Direct differentiation then yields

$$
\frac{1}{2} p^{\prime}(0)=-\frac{\alpha}{f(a)}+\frac{1}{a}+\alpha \overline{f(a)}
$$

and

$$
\frac{1}{8}\left(p^{\prime \prime}(0)-p^{\prime}(0)^{2}\right)=\left[-\frac{a_{2}}{f(a)}+\frac{\alpha^{2}}{f(a)^{2}}\left(\frac{f(a)}{\alpha a}-1\right)\right]\left(1-|f(a)|^{2}\right) .
$$

By using the inequality (2.2) of Lemma 2.1, we have

$\left|-\frac{a_{2}}{f(a)}+\frac{\alpha^{2}}{f(a)^{2}}\left(\frac{f(a)}{\alpha a}-1\right)\right|\left(1-|f(a)|^{2}\right) \leq \frac{1}{2}\left(1-\left|-\frac{\alpha}{f(a)}+\frac{1}{a}+\alpha \overline{f(a)}\right|^{2}\right)$.

That is,

$$
\begin{aligned}
& \left|a_{2} f(a)-\alpha^{2}\left(\frac{f(a)}{\alpha a}-1\right)\right| \\
& \quad \leq \frac{1}{2\left(1-|f(a)|^{2}\right)}\left(|f(a)|^{2}-\left|\frac{f(a)}{a}-\alpha\left(1-|f(a)|^{2}\right)\right|^{2}\right) .
\end{aligned}
$$

This implies

$$
\begin{aligned}
\operatorname{Re}\left\{a_{2} f(a)\right\} \geq & \alpha^{2} \operatorname{Re}\left\{\frac{f(a)}{\alpha a}-1\right\} \\
& -\frac{1}{2\left(1-|f(a)|^{2}\right)}\left(|f(a)|^{2}-\left|\frac{f(a)}{a}-\alpha\left(1-|f(a)|^{2}\right)\right|^{2}\right)
\end{aligned}
$$




$$
\begin{aligned}
& =\frac{1}{2}\left|\frac{f(a)}{a}\right|^{2} \frac{1-|a|^{2}}{1-|f(a)|^{2}}-\frac{1}{2} \alpha^{2}\left(1+|f(a)|^{2}\right) \\
& >-\frac{1}{2} \alpha^{2}\left(1+|f(a)|^{2}\right)>-\alpha^{2} .
\end{aligned}
$$

Note that for $k_{\alpha}(z)$, the infimum of $\operatorname{Re}\left\{a_{2} k_{\alpha}(z) / \alpha^{2}\right\}$ over $z \in \mathbb{D}$ is $-\left(1-\alpha^{2}\right)$. Thus, the constant -1 in Theorem 4.4 is best possible. This constant seems to be quite different from that for euclidean convex functions $f(z)=z+a_{2} z^{2}+\ldots$, in which case Fournier, Ma and Ruscheweyh [2] proved that $\operatorname{Re}\left\{a_{2} f(z)\right\}>-1 / 2$ for all $z \in \mathbb{D}$.

5. Schwarzian derivative of hyperbolically convex functions. Mejia and Pommerenke [6] investigated the Schwarzian derivative for hyperbolically convex functions. They conjectured that

$$
\begin{aligned}
& \sup \left\{\left(1-|z|^{2}\right)^{2}\left|S_{f}(z)\right|: f \text { is hyperbolically convex, } z \in \mathbb{D}\right\} \\
& \quad=\sup \{\sigma(\alpha): 0<\alpha \leq 1\} \approx 2.384,
\end{aligned}
$$

where

$$
\sigma(\alpha)=\max \left\{\left|S_{f}(0)\right|: f(z)=\alpha z+\ldots \text { is hyperbolically convex }\right\} .
$$

They also proved that $\sigma(\alpha) \leq 3\left(1-\alpha^{4}\right)$ for $e^{-2} \leq \alpha \leq 1$. In this section, we use the two-variable characterization to get a uniform upper bound on $\sigma(\alpha)$. Precisely, we establish an inequality for hyperbolically convex functions that is similar to the invariant form of the Trimble inequality for euclidean convex functions [10].

THEOREM 5.1. Suppose $f(z)$ (not necessarily normalized) is holomorphic and locally univalent in $\mathbb{D}$ with $f(\mathbb{D}) \subset \mathbb{D}$. Then $f(z)$ is hyperbolically convex if and only if

$$
\left(1-|z|^{2}\right)^{2}\left|S_{f}(z)\right|+\frac{3}{4}\left|\frac{D_{h 2} f(z)}{D_{h 1} f(z)}\right|^{2} \leq 3 .
$$

Proof. First, we assume that $f(z)$ is hyperbolically convex. It is enough to prove the desired inequality at any fixed $a \in \mathbb{D}$. Define

$$
p(z)=\frac{2 z f^{\prime}(z)}{f(z)-f(a)}-\frac{z+a}{z-a}+\frac{2 \overline{f(a)} z f^{\prime}(z)}{1-\overline{f(a)} f(z)} .
$$

Theorem 3.1 tells us that $p(z) \in \mathcal{P}$. Long but straightforward calculations result in

$$
\begin{aligned}
p^{\prime}(z)= & \frac{2\left(f^{\prime}(z)+z f^{\prime \prime}(z)\right)(f(z)-f(a))-2 z f^{\prime}(z)^{2}}{(f(z)-f(a))^{2}}+\frac{2 a}{(z-a)^{2}} \\
& +\frac{2 \overline{f(a)}}{(1-\overline{f(a)} f(z))^{2}}\left[\left(f^{\prime}(z)+z f^{\prime \prime}(z)\right)(1-\overline{f(a)} f(z))+\overline{f(a)} z f^{\prime}(z)^{2}\right]
\end{aligned}
$$


and

$$
\begin{aligned}
\lim _{z \rightarrow a} p^{\prime}(z)= & \frac{2}{3} a \frac{f^{\prime \prime \prime}(a)}{f^{\prime}(a)}-\frac{1}{2} a\left(\frac{f^{\prime \prime}(a)}{f^{\prime}(a)}\right)^{2}+\frac{f^{\prime \prime}(a)}{f^{\prime}(a)} \\
& +\frac{2 \overline{f(a)} f^{\prime}(a)}{\left(1-|f(a)|^{2}\right)}\left[1+\frac{a f^{\prime \prime}(a)}{f^{\prime}(a)}+\frac{\overline{f(a)} a f^{\prime}(a)}{1-|f(a)|^{2}}\right] .
\end{aligned}
$$

From the limits of $p(z)$ and $p^{\prime}(z)$, we can easily see that

$$
\lim _{z \rightarrow a}\left(2 z p^{\prime}(z)+1-p(z)^{2}\right)=\frac{4}{3} a^{2} S_{f}(a) .
$$

By using the inequality (2.1) of Lemma 2.1 and letting $z \rightarrow a$, we have

$$
|a|^{2}\left|\frac{4}{3} S_{f}(a)\right|+\left|1+\frac{a f^{\prime \prime}(a)}{f^{\prime}(a)}+\frac{2 \overline{f(a)} a f^{\prime}(a)}{1-|f(a)|^{2}}-\frac{1+|a|^{2}}{1-|a|^{2}}\right|^{2} \leq\left(\frac{2|a|}{1-|a|^{2}}\right)^{2} .
$$

As

$$
\left(1-|a|^{2}\right)^{2}\left|1+\frac{a f^{\prime \prime}(a)}{f^{\prime}(a)}+\frac{2 \overline{f(a)} a f^{\prime}(a)}{1-|f(a)|^{2}}-\frac{1+|a|^{2}}{1-|a|^{2}}\right|^{2}=\left|\frac{a D_{h 2} f(a)}{D_{h 1} f(a)}\right|^{2},
$$

we get

$$
|a|^{2}\left(1-|a|^{2}\right)^{2}\left|\frac{4}{3} S_{f}(a)\right|+\left|\frac{a D_{h 2} f(a)}{D_{h 1} f(a)}\right|^{2} \leq 4|a|^{2},
$$

which implies the desired inequality.

On the other hand, the inequality yields $\left|D_{h 2} f(z) / D_{h 1} f(z)\right| \leq 2$. Corollary 3.2 then implies that $f(z)$ is hyperbolically convex.

Corollary 5.2. Let $f(z)=\alpha z+a_{2} z^{2}+a_{3} z^{3}+\ldots$ be hyperbolically convex in $\mathbb{D}$. Then

$$
\left|\frac{a_{3}}{\alpha}-\left(\frac{a_{2}}{\alpha}\right)^{2}\right|+\frac{1}{2}\left|\frac{a_{2}}{\alpha}\right|^{2} \leq \frac{1}{2} .
$$

REMARK 5.1. Note that for the function $k_{\alpha}(z)$,

$$
\left|\frac{a_{3}}{\alpha}-\left(\frac{a_{2}}{\alpha}\right)^{2}\right|+\frac{1}{2}\left|\frac{a_{2}}{\alpha}\right|^{2}=\frac{1}{2}\left(1-\alpha^{4}\right) .
$$

Thus the constant $1 / 2$ in Corollary 5.2 is best possible. And from Theorem 5.1, we see that $\sup \{\sigma(\alpha): 0<\alpha \leq 1\} \leq 3$.

6. Distortion theorems. Finding sharp bounds on $\left|f^{\prime}(z)\right|$ for hyperbolically convex functions is not an easy problem. We even do not know the sharp order of growth for $\left|f^{\prime}(z)\right|$. In this section, we use the sharp growth theorem obtained in [3] to get the sharp lower bound on $\left|f^{\prime}(z)\right|$ for all $z \in \mathbb{D}$ and the sharp upper bound on $\left|f^{\prime}(z)\right|$ when $|z| \leq \sqrt{2}-1$. 
TheOREM 6.1. Let $f(z)=\alpha z+a_{2} z^{2}+\ldots$ be hyperbolically convex. Then for all $z \in \mathbb{D}$,

and for $|z| \leq \sqrt{2}-1$,

$$
\left|f^{\prime}(z)\right| \geq k_{\alpha}^{\prime}(-|z|)
$$

$$
\left|f^{\prime}(z)\right| \leq k_{\alpha}^{\prime}(|z|)
$$

Proof. In [3], we obtained the sharp growth theorem $-k_{\alpha}(-|z|) \leq$ $|f(z)| \leq k_{\alpha}(|z|)$ for hyperbolically convex $f(z)=\alpha z+a_{2} z^{2}+\ldots$ That is, if $f(z)$ is hyperbolically convex with $f(0)=0$, then

$$
\begin{aligned}
& \frac{2\left|f^{\prime}(0)\right||z|}{1+|z|+\sqrt{(1+|z|)^{2}-4\left|f^{\prime}(0)\right|^{2}|z|}} \\
& \quad \leq|f(z)| \leq \frac{2\left|f^{\prime}(0)\right||z|}{1-|z|+\sqrt{(1-|z|)^{2}+4\left|f^{\prime}(0)\right|^{2}|z|}}
\end{aligned}
$$

since $e^{-i \theta} f(z)$, where $\theta=\arg \left\{f^{\prime}(0)\right\}$, is also hyperbolically convex. By using the inequalities above for

$$
f_{a}(z)=\frac{f((z+a) /(1+\bar{a} z))-f(a)}{1-\overline{f(a)} f((z+a) /(1+\bar{a} z))}
$$

we see that

$$
\begin{aligned}
& \frac{2\left|D_{h 1} f(a)\right||z|}{1+|z|+\sqrt{(1+|z|)^{2}-4\left|D_{h 1} f(a)\right|^{2}|z|}} \\
& \leq\left|\frac{f((z+a) /(1+\bar{a} z))-f(a)}{1-\overline{f(a)} f((z+a) /(1+\bar{a} z))}\right| \leq \frac{2\left|D_{h 1} f(a)\right||z|}{1-|z|+\sqrt{(1-|z|)^{2}+4\left|D_{h 1} f(a)\right|^{2}|z|}} .
\end{aligned}
$$

If we let $z=-a$, we get

$$
|f(a)| \leq \frac{2\left|D_{h 1} f(a)\right||a|}{1-|a|+\sqrt{(1-|a|)^{2}+4\left|D_{h 1} f(a)\right|^{2}|a|}}
$$

and

$$
|f(a)| \geq \frac{2\left|D_{h 1} f(a)\right||a|}{1+|a|+\sqrt{(1+|a|)^{2}-4\left|D_{h 1} f(a)\right|^{2}|a|}} .
$$

The inequality (6.1) can be rewritten as

$$
|f(a)| \sqrt{(1-|a|)^{2}+4\left|D_{h 1} f(a)\right|^{2}|a|} \leq 2\left|D_{h 1} f(a)\right||a|-(1-|a|)|f(a)|,
$$

which is equivalent to

$$
\left(|a|-|f(a)|^{2}\right)\left|D_{h 1} f(a)\right| \geq(1-|a|)|f(a)| .
$$

Thus,

$$
\left|f^{\prime}(a)\right| \geq \frac{|f(a)|\left(1-|f(a)|^{2}\right)}{(1+|a|)\left(|a|-|f(a)|^{2}\right)}
$$


We want to show that the right side is an increasing function of $|f(a)|$ when $0 \leq|f(a)| \leq|a| \leq 1$. The function $h(x)=x\left(1-x^{2}\right) /\left(|a|-x^{2}\right), 0 \leq x \leq|a|$ $\leq 1$, satisfies

$$
\begin{aligned}
\left(|a|-x^{2}\right)^{2} h^{\prime}(x) & =|a|+(1-3|a|) x^{2}+x^{4} \\
& =\left(|a|-x^{2}\right)^{2}+\left(|a|+x^{2}\right)(1-|a|),
\end{aligned}
$$

which is clearly nonnegative when $0 \leq x \leq|a| \leq 1$. Therefore, $h^{\prime}(x) \geq 0$ when $0 \leq x \leq|a| \leq 1$ and so the right side of (6.3) is an increasing function of $|f(a)|$. Since $-k_{\alpha}(-|a|) \leq|f(a)|$, we have

$$
\left|f^{\prime}(a)\right| \geq \frac{-k_{\alpha}(-|a|)\left(1-k_{\alpha}^{2}(-|a|)\right)}{(1+|a|)\left(|a|-k_{\alpha}^{2}(-|a|)\right)}=k_{\alpha}^{\prime}(-|a|) .
$$

Now we verify the upper bound. First we derive

$$
\left|f^{\prime}(a)\right| \leq \frac{|f(a)|\left(1-|f(a)|^{2}\right)}{(1-|a|)\left(|a|+|f(a)|^{2}\right)} .
$$

The inequality (6.2) can be written as

$$
2\left|D_{h 1} f(a)\right||a|-(1+|a|)|f(a)| \leq|f(a)| \sqrt{(1+|a|)^{2}-4\left|D_{h 1} f(a)\right|^{2}|a|} .
$$

If $2\left|D_{h 1} f(a)\right||a|-(1+|a|)|f(a)| \leq 0$, then

$$
\left|f^{\prime}(a)\right| \leq \frac{|f(a)|\left(1-|f(a)|^{2}\right)}{2|a|(1-|a|)} \leq \frac{|f(a)|\left(1-|f(a)|^{2}\right)}{(1-|a|)\left(|a|+|f(a)|^{2}\right)} .
$$

If $2\left|D_{h 1} f(a)\right||a|-(1+|a|)|f(a)| \geq 0$, then by squaring both sides we get

$$
\left(|a|+|f(a)|^{2}\right)\left|D_{h 1} f(a)\right| \leq(1+|a|)|f(a)| .
$$

This is the same as (6.4).

Second, we show that the right side of (6.4) is an increasing function of $|f(a)|$ when $|a| \leq \sqrt{2}-1$. The function $l(x)=x\left(1-x^{2}\right) /\left(|a|+x^{2}\right)$, $0 \leq x \leq|a|$, satisfies $\left(|a|+x^{2}\right)^{2} l^{\prime}(x)=|a|-(1+3|a|) x^{2}-x^{4}$, which is nonnegative if

$$
x^{2} \leq \frac{\sqrt{(1+3|a|)^{2}+4|a|}-(1+3|a|)}{2} .
$$

Direct calculation shows that when $|a| \leq \sqrt{2}-1$,

$$
|a|^{2} \leq \frac{\sqrt{(1+3|a|)^{2}+4|a|}-(1+3|a|)}{2} .
$$

Thus, $l^{\prime}(x) \geq 0$ when $0 \leq x \leq|a| \leq \sqrt{2}-1$.

Finally, as $|f(a)| \leq k_{\alpha}(|a|)$, we obtain

$$
\left|f^{\prime}(a)\right| \leq \frac{k_{\alpha}(|a|)\left(1-k_{\alpha}^{2}(|a|)\right)}{(1-|a|)\left(|a|+k_{\alpha}^{2}(|a|)\right)}=k_{\alpha}^{\prime}(|a|) .
$$

Recently, Mejia, Pommerenke and Vasil'ev [5] got the same upper bound on $\left|f^{\prime}(z)\right|$ when $|z| \leq \sqrt{2}-1$ by using a somewhat different method, although 
their approach also resulted in the same inequality (6.4). We included our proof since the method for the upper and lower bounds is the same. Also note from our proof that $\left|f^{\prime}(a)\right| \leq k_{\alpha}^{\prime}(|a|)$ holds as long as

$$
|f(a)|^{2} \leq \frac{\sqrt{(1+3|a|)^{2}+4|a|}-(1+3|a|)}{2} .
$$

Since $|f(a)| \leq k_{\alpha}(|a|)$, the upper bound in Theorem 6.1 is valid for all $a$ that satisfy

$$
k_{\alpha}(|a|)^{2} \leq \frac{\sqrt{(1+3|a|)^{2}+4|a|}-(1+3|a|)}{2} .
$$

\section{References}

[1] L. V. Ahlfors, Complex Analysis, 3rd ed., McGraw-Hill, New York, 1979.

[2] R. Fournier, J. Ma and S. Ruscheweyh, Convex univalent functions and omitted values, preprint.

[3] W. Ma and D. Minda, Hyperbolically convex functions, Ann. Polon. Math. 60 (1994), 81-100.

[4] - - - Hyperbolic linear invariance and hyperbolic k-convexity, J. Austral. Math. Soc. Ser. A 58 (1995), 73-93.

[5] D. Mejia, Ch. Pommerenke and A. Vasil'ev, Distortion theorems for hyperbolically convex functions, preprint.

[6] D. Mejia and Ch. Pommerenke, On hyperbolically convex functions, J. Geom. Anal., to appear.

[7] S. Ruscheweyh, Convolutions in Geometric Function Theory, Les Presses de l'Université de Montréal, Montréal, 1982.

[8] T. Sheil-Small, On convex univalent functions, J. London Math. Soc. 1 (1969), 483-492.

[9] T. J. Suffridge, Some remarks on convex maps of the unit disk, Duke Math. J. 37 (1970), 775-777.

[10] S. Y. Trimble, A coefficient inequality for convex univalent functions, Proc. Amer. Math. Soc. 48 (1975), 266-267.

School of Integrated Studies

Pennsylvania College of Technology

Williamsport, Pennsylvania 17701

U.S.A.

E-mail:wma@pct.edu
Department of Mathematical Sciences University of Cincinnati Cincinnati, Ohio 45221-0025

U.S.A.

E-mail: David.Minda@math.uc.edu 